JORGE J. GUMUCIO, EDITOR

Department of Internal Medicine

Division of Gastroenterology (111D)

VA Medical Center/University of Michigan Ann Arbor, Michigan 48105

\section{LESSONS FROM THE REPRESSION OF THE a-FETOPROTEIN GENE IN THE ADULT LIVER}

Emerson JA, Vacher J, Cirillo LA, Tilghman SM, Tyner $A L$. The zonal expression of $\alpha$-fetoprotein transgenes in the livers of adult mice. Dev Dyn 1992;195:55-66.

\section{ABSTRACT}

The developmental regulation of the $\alpha$-fetoprotein (AFP) gene in liver results in high-level expression in the fetus, followed by dramatic transcriptional repression after birth. We have examined the mouse AFP gene for transcriptional control sequences that may be involved in its postnatal repression in liver. We showed previously that removal of a DNA region between positions -250 base pairs (bp) and -838 bp of the AFP gene resulted in the persistence of expression of an AFP minigene in the postpartum liver of transgenic mice (Vacher and Tilghman, Science 250;1732-1735, 1990). This study examines the distribution of these transgene transcripts in liver using in situ hybridization. We show that there is a zonal distribution of minigene transcripts in the adult livers of these animals. Hepatocytes surrounding the central veins express high levels of minigene transcripts, while hepatocytes in the intermediate and portal areas contain few, if any, transcripts. Quantitative RNAse protection analysis shows a decrease in transgene RNA levels after birth, consistent with repression in all but a small subset of hepatocytes. These results indicate that repression in the pericentral hepatocytes is dependent upon the presence of a cis-acting, negative-regulatory domain, which is located between the enhancers and the proximal promoter of the AFP gene. In contrast, this domain is not essential for complete repression of AFP transgenes in the intermediate zone and periportal hepatocytes.

\section{COMMENTS}

$\alpha$-Fetoprotein (AFP) is a $72-\mathrm{kD}$ protein expressed in the fetal liver and yolk sac $(1,2)$. Despite its low, almost undetectable levels in the adult liver, this protein has provoked more research and clinical interest than most other genes expressed in liver. AFP is detectable in human fetal serum at approximately $10 \mathrm{wk}$ of gestation, about the time when endodermal cells from the ventral foregut meet the cardiac mesenchyma and form the liver primordium. In rodents, days 9.5 to 10 of gestation seem to be the time at which expression of liver-specific genes starts; albumin mRNA is also detectable at this point (3, 4).

HEPATOL,OGY 1993;18:1009-1016. $\mathbf{3 1 / 8 / 4 9 8 4 1}$
The albumin and AFP genes have much in common, including $39 \%$ overall sequence homology. Each of the genes encoding these two proteins in mammals exists as a single copy and arose from the duplication of an ancestral gene 300 to 500 million years ago. These genes remained linked throughout this period, with a $14-\mathrm{kb}$ distance between the $3^{\prime}$ terminus of albumin gene and the 5 ' terminus of the AFP gene $(1,2,5)$. The expression of both genes is tissue specific and predominantly controlled at the transcriptional level. In fetal liver, albumin and AFP transcription seem to be activated in a coordinate fashion. However, after birth these two genes seem to be regulated independently. Thus albumin transcription increases, whereas AFP gene transcription is dramatically reduced. An approximate 10,000-fold reduction in AFP mRNA level occurs after birth. Another example of independent regulation is the finding that AFP transcription increases during carcinogenesis and in the regenerating liver after toxic injury, whereas transcription of the albumin gene is not altered substantially in either condition.

The precise function of AFP, the most abundant protein in fetal plasma, is not known $(1,2)$. There is no established in vivo model such as the analbuminemic rat with which to study whether the function of AFP is vital in fetal life. It has been proposed that AFP is involved in the maintenance of fetal oncotic pressure and in binding and delivery of fetal hormones, bilirubin and organic cations. It has also been suggested that AFP facilitates the immunosuppressive mechanisms that protect the fetoplacental unit from rejection. Despite our lack of comprehension of the AFP function in fetal liver, work on AFP gene regulation has provided important clues to our understanding of the tissue-specific expression of genes during liver development.

After the cloning and characterization of the AFP gene, studies were concentrated on the characterization of the sequences involved in the regulation of the postnatal transcriptional repression of this gene $(1,2,5)$. Two alternatives have been proposed to explain the repression phenomenon. The first assumes the presence of positive factor(s) necessary for AFP transcription activation that are inactivated after birth. The second is the presence of a postnatal repressor or negative regulator of AFP transcription. To identify which of these alternatives is applicable to AFP gene regulation, Tilghman and coworkers studied the sequences in the AFP gene that respond to positive and negative regulators. They performed a series of experiments in which foreign genes containing deletions of the 5 ' flanking region of AFP were introduced into hepatoma cells. By comparing the transcriptional activity of each of these 


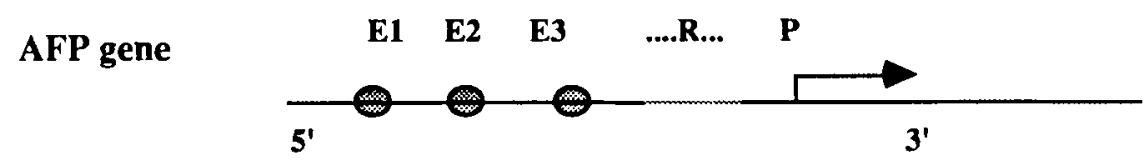

\section{Minigene Constructs}

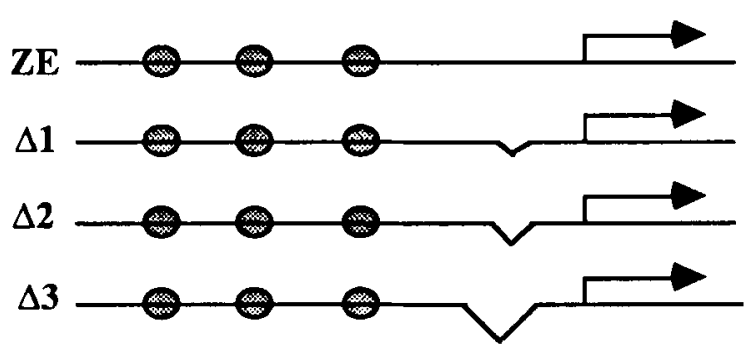

Fig. 1. (Top) The wild-type AFP gene has three enhancers $(E 1, E 2$ and $E 3)$ and a promoter $(P)$. The region represented with a dotted line $(R)$ represents an area in which repressor elements are presumed to be present. (Bottom) The constructs used to prepare transgenic mice are represented. A minigene containing the promoter region and a small portion of the open reading frame was created to prepare the constructs. Serial deletions were made at regions located between the enhancers and the minigene. $Z E=$ construct with no deletions; $\Delta 1=$ construct with deletion from -838 to $-604 \mathrm{bp} ; \Delta 2=$ construct with a deletion from -838 to $-250 \mathrm{bp} ; \Delta 3=$ construct with a deletion from -838 to $-118 \mathrm{bp}$.

sequences, the authors were able to characterize three "enhancer" elements and a promoter. The enhancer elements, located $6.5,5$ and $2.5 \mathrm{~kb}$ upstream of the AFP promoter, were important for the activation of the AFP gene and acted in a position-and orientation-independent manner (Fig. 1). Studies with cell cultures have shown that although each of these elements is an important modulator of the transcriptional activity of the AFP gene, the enhancers do not seem to regulate tissue specificity. However, studies using in vitro cell culture systems have been limited by the use of transformed cells, which probably lack the normal cell-to-cell interactions and growth patterns.

To circumvent these problems the same group took advantage of an in vivo system, the transgenic mouse (2). In this method, DNA constructs consisting of a portion of the 5' flanking region of the gene of interest are attached to a promoter and a reporter gene (in this case, an internally deleted structural gene called a "minigene"). These constructs are then introduced into the germ line of mice. The founders are selected on the basis of the integration of these constructs into the genome and on the expression of this "transgene" in the tissue of interest. This technology allowed the investigators to prove that, in fetal liver, none of the three enhancers was crucial in the regulation of the tissuespecific expression of the AFP gene. However, the enhancers were important in activating transcription. The replacement of the AFP enhancers with those of the albumin gene did not seem to alter the inhibition of transcription observed after birth. Therefore these enhancers were not responsible for the developmental repression of the AFP gene.

Using tissue culture methodology and transgenic mice, the same group showed that the proximal $1 \mathrm{~kb}$ of the $5^{\prime}$ flanking region had sequences that were responsible for the transcriptional decline of AFP after birth. When a region between -250 and $-838 \mathrm{bp}$ was removed, the AFP expression was not repressed in adult life. Transgenic lines prepared with three constructs carrying different deletions between -250 and -838 bp (Fig. 1) exhibited disturbances in the repression of the AFP mRNA. These sequences were, therefore, involved in the postnatal repression of AFP. These sequences represent the first defined repressor(s) which has a functional significance in vivo (2). Of interest was that mRNA from all three constructs was expressed in liver and yolk sac in fetal life, suggesting that the deleted sequences were not the domains responsible for the tissue-specific expression of the AFP gene.

In this study, Emerson et al. expanded these observations and provided a new dimension of AFP regulation in liver. Using the same transgenic lines, they examined the cellular distribution of the AFP mRNA in liver. Their results were quite interesting. Initially, using animals carrying a construct containing the intact AFP gene, the authors investigated the distribution of AFP mRNA at different stages of development (construct ZE in Fig. 1). In fetal life, AFP mRNA was homogeneously distributed among all hepatocytes in the liver cell plate. However, starting from the first postnatal week, the AFP mRNA was restricted to the perivenous area. Two weeks after birth, only one or two rows of hepatocytes surrounding the hepatic venule expressed AFP mRNA. From then on, AFP mRNA disappeared completely. Thus the transcriptional decline of the AFP gene in normal liver, as detected with in situ hybridization, corresponds to a decrease in the number of hepatocytes transcribing this gene. Furthermore, the hepatocytes transcribing the AFP gene were perivenular. Thus, there seems to be both a temporal and a hepatocyte position-dependent repression of the AFP gene.

Next, the livers of the animals carrying deletions in this $5^{\prime}$ flanking region of the AFP gene (-250 and -838 bp; $\Delta 3$ and $\Delta 7$ constructs in Fig. 1) were studied. Strikingly, in these experiments, one or two rows of cells surrounding the hepatic venule continued to express AFP in adult life. Thus repression of AFP in perivenular 
cells was blocked by the deletion of this 588-bp fragment $(-250$ to $-838 \mathrm{bp})$ in the AFP gene. The authors concluded that this sequence must contain a negative regulatory domain necessary and sufficient for the complete repression of the AFP gene transcription. Another interesting observation was the delay observed in the gradual disappearance of the AFP transcripts from all other hepatocytes (periportal and intermediate). The animals carrying $\Delta 3$ constructs repressed AFP expression with a 4-wk delay, compared with animals carrying ZE constructs. This finding suggests that the deleted sequences in the $\Delta 3$ construct have a complementary role in the postnatal repression of AFP in hepatocytes located in the periportal and intermediate areas in the cell plate.

This work therefore identifies AFP as the first mammalian gene with intragenic repressor sequences. These cis-acting repressor sequences are functional only in a subpopulation of perivenular hepatocytes and only at a specific point in development. Interestingly, in rodents, this phase corresponds to the completion of the anatomical development of liver structure and to the establishment of the adult pattern of expression of certain genes such as those involved in ammonia metabolism. In contrast, the hepatocytes that are not in a perivenular location seem to have the ability to repress the expression of AFP. Most likely, other sequences have a primary role in AFP repression in nonperivenular hepatocytes.

As mentioned previously, AFP gene expression can be repressed by chemical injury or carcinogenesis, and the common denominator in these events seems to be induction of cell proliferation. However, AFP is not markedly induced in other models of cell proliferation such as partial hepatectomy $(8,9)$. In addition, agents such as $\mathrm{CCl}_{4}$ that induce AFP cause perivenular hepatocyte damage. Therefore it is possible that AFP expression is induced not just by cell damage or activation of hepatocyte proliferation but by the disappearance of trans-acting repressor elements located in perivenular hepatocytes. After $\mathrm{CCl}_{4}$ damage, glutamine synthetase expression (as shown at the mRNA and the apoprotein level), disappears as soon as the perivenular cells are damaged (10). Expression reappears as soon as appropriate cell contacts are established. It has been suggested that the interaction between perivenular hepatocytes (endoderm derived) and hepatic venule (of mesenchymal origin) in the perivenular area is responsible for the generation of signals or trans-acting elements that bind to the -838 to $-250 \mathrm{bp}$ sequence in AFP gene.

It has been shown in the past decade that perivenular hepatocytes differ from the rest of the hepatocytes in the liver cell plate in several respects (6). First, perivenular hepatocytes are located at the end of the liver cell plate and thus are exposed to lower concentrations of nutrients, hormones and oxygen. Second, certain genes are only expressed in this compartment, including glutamine synthetase, ornithine aminocarboxylase, and $\alpha 2 \mathrm{u}$-globulin $(3,6)$. Third, perivenular hepatocytes are in close proximity to the hepatic venule. It has been proposed that the regulation of certain genes in these perivenular hepatocytes depends on cell-to-cell interactions involving the cells of the hepatic venule. This proposal remains to be proved. However, perivenular hepatocytes transplanted into the spleen lose the ability to transcribe glutamine synthetase when they are not in close proximity to vascular structures. Periportal hepatocytes, in close contact with splenic vessels, acquire the ability to express glutamine synthetase $(7,10)$.

This article, like any other good study, raises more questions than it answers. The study highlights the importance of looking at the tissue distribution of genes while investigating the developmental and positional repression of a model gene. Future work in this area will improve our understanding of the position-dependent control of gene expression in the liver cell plate.

BAHRI M. BILIR, M.D.
Division of Gastroenterology
University of Michigan Medical Center
Veterans Affairs Medical Center
Ann Arbor, Michigan 48105

\section{REFERENCES}

1. Camper SA, Godbout R, Tilghman SM. The developmental regulation of albumin and alpha feto protein gene expression. Prog Nucleic Acid Res Mol Bio 1989;36:131-143.

2. Vasher J, Tilghman SM. Dominant negative regulation of the mouse alpha-feto protein gene in adult liver. Science 1990;250: 1732-1735.

3. Kuo FC, Paulson KE, Darnell JE. Positional and developmental regulation of glutamine synthetase expression in mouse liver. Mol Cell Biol 1988;8:4966-4971.

4. Cascio S, Zaret KJ. Hepatocyte differentiation initiates during endodermal-mezenchymal interactions prior to liver formation. Development 1991;113:217-225.

5. Spear BT, Tilghman SM. Role of alpha-feto protein regulatory elements in transcriptional activation in transient heterokaryons. Mol Cell Biol 1990;10:5047-5054.

6. Gumucio JJ. Hepatocyte heterogeneity: the coming of age, from the description of a biological curiosity to a partial understanding of its physiological meaning and regulation. HEPATOLOGY 1989;9: 154-160.

7. Lamers WH, Been W, Charles R, Moorman AFM. Hepatocytes explanted in the spleen preferentially express carbamoylphosphate synthetase rather than glutamine synthetase. HePATOLOGY 1990;12:702-707.

8. Tournier I, Legres L, Schoevaert D, Feldmann G, Bernuau D. Cellular analysis of alpha fetoprotein gene activation during carbon tetracholoride and D-galactosamine induced acute liver injury in rats. Lab Invest 1988;59:657-665.

9. Petropoulos CJ, Yaswen P, Panzina M, Fausto N. Cell lineages in liver carcinogenesis: possible clues from the studies of the distribution of alpha-feto protein RNA sequences in cell populations isolated from normal, regenerating and preneoplastic rat livers. Cancer Res 1985;45:5762-5768.

10. Kuo FC, Darnell JE Jr. Evidence that interaction of hepatocytes with the collecting (hepatic) veins triggers position specific transcription of the glutamine synthetase and ornithine amino transferase genes in mouse liver. Mol Cell Biol 1991;11:6050-6058.

\section{CHASING THE ESCAPE MUTANT}

Waters JA, Kennedy $M$, Voet $P$, Hauser P, Petre J, Carman W, Thomas HC. Loss of the common " $a$ " determinant of hepatitis B surface antigen by a vaccine- 